\title{
Parametric Resonance in Mechanics: Classical Problems and New Results*
}

\author{
Alexander P. SEYRANIAN** \\ ${ }^{* *}$ Institute of Mechanics \\ Moscow State Lomonosov University \\ Michurynski pr.1, Moscow 119192, Russia \\ e-mail: seyran@imec.msu.ru
}

\begin{abstract}
We formulate and solve parametric resonance problems for one- and multiple degrees of freedom systems in three-dimensional space of physical parameters: excitation frequency, amplitude, and viscous damping coefficient assuming that the last two parameters are small. The main result obtained here is that we find the instability domains (simple and combination parametric resonances) as half-cones in three-parameter space with the use of eigenfrequencies and eigenmodes of the corresponding conservative system.
\end{abstract}

Key words: Parametric Resonance, Mechanical Problems, Instability Domains

\section{Introduction}

This work is devoted to stability problems of periodic systems dependent on multiple constant parameters. This subject has been a challenge for more than one hundred years since Mathieu, Floquet, Hill, Rayleigh, Lyapunov, Poincare. From the very beginning these problems were multiparameter. We formulate and solve parametric resonance problems for one- and multiple degrees of freedom systems in three-dimensional space of physical parameters: excitation frequency, amplitude, and viscous damping coefficient assuming that the last two parameters are small. The main result obtained here is that we find the instability domains (simple and combination parametric resonances) as half-cones in three-parameter space with the use of eigenfrequencies and eigenmodes of the corresponding conservative system.

In Section 1 derivatives of a monodromy (Floquet) matrix with respect to problem parameters are presented. They are important for description of behavior of monodromy matrices in the vicinity of an initial point in parameter space, and hence the stability study. The derived relations allow to find the derivatives of simple multipliers, responsible for stability of the system with respect to parameters, and predict their behavior with a change of parameters. In Section 2 resonance domains for Hill's equation with damping are investigated. It is shown that they represent halves of cones in three-parameter space. In Section 3 another classical problem of finding instability regions for a system with periodically varying moment of inertia is formulated and solved. An equation describing small torsional oscillations of the system with periodic coefficients dependent on four constant parameters including damping is derived. Analytical results for instability (parametric resonance) regions in parameter space are obtained and numerical examples are presented. In Section 4 a problem of stabilization of a vertical (inverted) position of a pendulum under high frequency vibration of the suspension point is considered. Small viscous damping is taken into account, and periodic excitation function describing vibration of the suspension point is assumed to be arbitrary. A formula for stability region of Hill's 
equation with damping near zero frequency is obtained. For several examples it is shown that analytical and numerical results are in a good agreement with each other. An asymptotic formula for stabilization region of the inverted pendulum with arbitrary periodic excitation function is derived. It is shown that the effect of small viscous damping is of the third order, and taking it into account leads to increasing critical stabilization frequency. In Section 5 another important application of damped Hill's equation is considered. It is connected with the study of stability of periodic motions in nonlinear dynamical systems. It is shown how to find stable and unstable regimes for harmonically excited Duffing's equation. Then, in Section 6 linear vibrational systems with arbitrary degrees of freedom with periodic coefficients depending on three independent parameters: frequency and amplitude of periodic excitation, and damping coefficient are considered with the assumption that the last two quantities are small. Instability of the trivial solution of the system (parametric resonance) is studied. Two important specific cases of excitation matrix are studied: a symmetric matrix and a stationary matrix multiplied by a scalar periodic function. It is shown that in both cases the resonance domains are halves of cones in the three-dimensional space with the boundary surface coefficients depending only on the eigenfrequencies, eigenmodes and system matrices. The obtained relations allow to analyze influence of growing eigenfrequencies and resonance number on instability domains. In Section 7 as a mechanical application Bolotin's problem of dynamic stability of a thin beam loaded by periodic bending moments is solved. Comparison between analytical asymptotic formulae and numerical results for resonance domains show that they are in a good agreement with each other.

This paper is a review of the results on parametric resonance obtained by the author with Pauli Pedersen and Frederik Solem (Denmark), Alexei A. Mailybaev and Andrei A. Seyranian (Russia), Hiroshi Yabuno and Koji Tsumoto (Japan), and Carlo Cattani (Italy), see the list of references below.

\section{Derivatives of the monodromy matrix with respect to parameters}

We consider a system of linear differential equations

$$
\dot{\mathbf{x}}=\mathbf{G x}
$$

where $\mathbf{G}=\mathbf{G}(t, \mathbf{p})$ is a real square matrix of dimension $m$, which is smoothly depending on a vector of real parameters $\mathbf{p}=\left(p_{1}, p_{2}, \ldots p_{n}\right)$ and is a continuous periodic function of the time $\mathbf{G}(t, \mathbf{p})=\mathbf{G}(t+T, \mathbf{p}), T$ being a period. We denote linear independent solutions of system (1) as $\mathbf{x}_{1}(t), \mathbf{x}_{2}(t), \ldots, \mathbf{x}_{m}(t)$ and form out of them a fundamental matrix $\mathbf{X}(t)=\left[\mathbf{x}_{1}(t), \mathbf{x}_{2}(t), \ldots, \mathbf{x}_{m}(t)\right]$. The $\mathbf{X}(t)$ matrix satisfying the equations

$$
\dot{\mathbf{X}}=\mathbf{G X}, \quad \mathbf{X}(0)=\mathbf{I}
$$

where $\mathbf{I}$ is the identity matrix of dimension $m$, is called a matriciant, and the matrix $\mathbf{F}=\mathbf{X}(T)$ is called a monodromy matrix.

According to the Floquet theory, see Nayfeh and Mook (1979) and Yakubovich and Starzhinskii (1987), stability of system (1.1) is determined by multipliers (eigenvalues of the monodromy matrix): if all the multipliers for their absolute value are less than one, the system is asymptotically stable, and if at least one of them is greater than one, the system becomes unstable.

Along with (1.2) we consider an adjoint system of equations for the matrix $\mathbf{Y}$

$$
\dot{\mathbf{Y}}=-\mathbf{G}^{\mathbf{T}} \mathbf{Y}, \quad \mathbf{Y}(0)=\mathbf{I}
$$

The solutions $\mathbf{X}(t)$ and $\mathbf{Y}(t)$ of equations (1.2) and (1.3) are related by the equality, see Yakubovich and Starzhinskii (1987)

allowing to express $\mathbf{Y}(t)=\left[\mathbf{X}^{\mathbf{T}}(t)\right]^{-1}$

$$
\mathbf{X}^{\mathbf{T}}(t) \mathbf{Y}(t)=\mathbf{I}
$$

Now we assume that the vector of parameters takes a variation $\mathbf{p}=\mathbf{p}_{0}+\Delta \mathbf{p}$. Hence, the $\mathbf{G}$ matrix, and therefore the matriciant $\mathbf{X}(t)$ obtain variations. This accordingly leads to a 
change of the monodromy matrix $\mathbf{F}$. The formulas for the first and second derivatives of a monodromy matrix with respect to parameters were derived in Seyranian et al. $(1999,2000)$ in the form of integrals over the period

$$
\begin{gathered}
\frac{\partial \mathbf{F}}{\partial p_{k}}=\mathbf{F}_{0} \int_{0}^{T} \mathbf{Y}_{0}^{\mathbf{T}} \frac{\partial \mathbf{G}}{\partial p_{k}} \mathbf{X}_{0} d t, \quad k=1, \ldots, n \\
\frac{\partial^{2} \mathbf{F}}{\partial p_{i} \partial p_{j}}=\mathbf{F}_{0}\left\{\int_{0}^{T} \mathbf{Y}_{0}^{\mathbf{T}} \frac{\partial^{2} \mathbf{G}}{\partial p_{i} \partial p_{j}} \mathbf{X}_{0} d t+\int_{0}^{T} \mathbf{Y}_{0}^{\mathbf{T}} \frac{\partial \mathbf{G}}{\partial p_{i}} \mathbf{X}_{0}\left(\int_{0}^{\tau} \mathbf{Y}_{0}^{\mathbf{T}} \frac{\partial \mathbf{G}}{\partial p_{j}} \mathbf{X}_{0} d \zeta\right) d \tau+\right. \\
\left.+\int_{0}^{T} \mathbf{Y}_{0}^{\mathbf{T}} \frac{\partial \mathbf{G}}{\partial p_{j}} \mathbf{X}_{0}\left(\int_{0}^{\tau} \mathbf{Y}_{0}^{\mathbf{T}} \frac{\partial \mathbf{G}}{\partial p_{i}} \mathbf{X}_{0} d \zeta\right) d \tau\right\}, i, j=1, \ldots, n
\end{gathered}
$$

where the zero subscript means that the corresponding value is taken at $\mathbf{p}=\mathbf{p}_{0}$. Note that to find derivatives (1.5) and (1.6) it is necessary to know only the matriciants $\mathbf{X}_{0}(t), \mathbf{Y}_{0}^{\mathbf{T}}(t)=\mathbf{X}_{0}^{-1}(t)$ and the derivatives of the $\mathbf{G}$ matrix with respect to the parameters taken at $\mathbf{p}=\mathbf{p}_{0}$. Using derivatives (1.5) and (1.6) a variation of the monodromy matrix can be given in the form

$$
\mathbf{F}\left(\mathbf{p}_{0}+\Delta \mathbf{p}\right)=\mathbf{F}_{0}+\sum_{k=1}^{n} \frac{\partial \mathbf{F}}{\partial p_{k}} \Delta p_{k}+\frac{1}{2} \sum_{i, j=1}^{n} \frac{\partial^{2} \mathbf{F}}{\partial p_{i} \partial p_{j}} \Delta p_{i} \Delta p_{j}+\cdots
$$

Knowing the derivatives of the monodromy matrix we can calculate the value of this matrix in the vicinity of the initial point $\mathbf{p}_{0}$, and therefore estimate behavior of the multipliers responsible for the stability of system (1.1) when the problem parameters are changed. The first and second derivatives of the monodromy matrix for the case when the period $T$ also depends on parameters were derived in Seyranian et al. (2000), and Seyranian and Mailybaev (2003) .

\section{Resonance domains for Hill's equation with damping}

In this section we consider Hill's equation with damping

$$
\ddot{y}+\beta \dot{y}+\left[\omega^{2}+\varepsilon \varphi(t)\right] y=0
$$

where $\beta$ is the damping coefficient, $\varepsilon$ is the excitation amplitude, $\omega$ is the natural frequency, and $\varphi(t)$ is a continuous periodic function of time with the period $2 \pi$. For the sake of simplicity we assume that the periodic function has zero mean value

$$
\int_{0}^{2 \pi} \varphi(t) d t=0
$$

Equation (2.1) has been considered by many authors, see Nayfeh and Mook (1979) and Merkin (1997).

Our aim is to find analytically the domains of instability of the trivial solution $y=0$ (the domains of parametric resonance) in the case of small excitation amplitude $\mathcal{E}$, damping coefficient $\beta$, and arbitrary natural frequency $\omega \neq 0$. For this purpose we represent equation (2.1) in the form (1.1)

$$
\dot{\mathbf{x}}=\mathbf{G}(\mathbf{p}, t) \mathbf{x}
$$

with

$$
\mathbf{x}=\left(\begin{array}{l}
y \\
\dot{y}
\end{array}\right), \quad \mathbf{G}(t, \mathbf{p})=\left[\begin{array}{cc}
0 & 1 \\
-\omega^{2}-\varepsilon \varphi(t) & -\beta
\end{array}\right]
$$

This system contains explicitly three parameters $\mathbf{p}=(\varepsilon, \beta, \omega)$. If we substitute 
$\varepsilon=0, \beta=0$ in (2.4) then it is easy to find the matriciant and its inverse from (1.2) and $(2.3),(2.4)$ as

$$
\mathbf{X}_{0}(t)=\left[\begin{array}{cc}
\cos \omega t & \omega^{-1} \sin \omega t \\
-\omega \sin \omega t & \cos \omega t
\end{array}\right], \quad \mathbf{X}_{0}^{-1}(t)=\left[\begin{array}{cc}
\cos \omega t & -\omega^{-1} \sin \omega t \\
\omega \sin \omega t & \cos \omega t
\end{array}\right]
$$

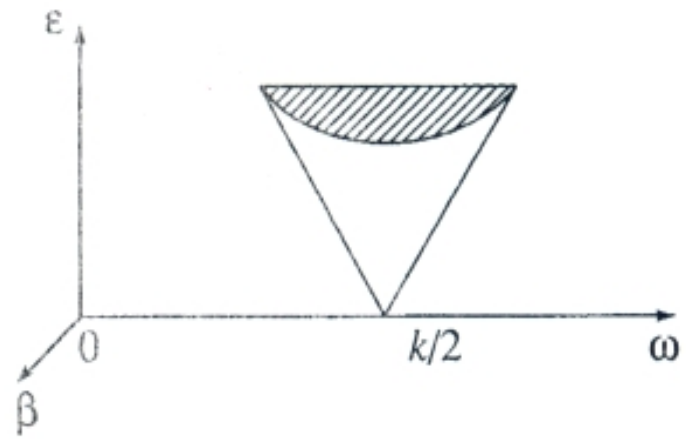

Figure 1: The instability domains for Hill's equation with damping.

So, when $\varepsilon=0, \beta=0$ we have the monodromy matrix in the form

$$
\mathbf{F}_{0}=\mathbf{X}_{0}(2 \pi)=\left[\begin{array}{cc}
\cos 2 \pi \omega & \omega^{-1} \sin 2 \pi \omega \\
-\omega \sin 2 \pi \omega & \cos 2 \pi \omega
\end{array}\right]
$$

The eigenvalues of this matrix (the multipliers) are

$$
\rho_{1,2}=\cos 2 \pi \omega \pm i \sin 2 \pi \omega
$$

If $\omega \neq k / 2, k=1,2, \ldots$ the multipliers are complex conjugate quantities lying on the unit circle (stability). We can show that when small periodic excitation and damping are added $(\varepsilon>0, \beta>0)$ then the multipliers move inside the unit circle which implies asymptotic stability. Indeed, in this case the multipliers due to continuity property and reality of the matriciant remain complex conjugate quantities. For the multipliers we have a quadratic equation

$$
\rho^{2}+c_{1} \rho+c_{2}=0
$$

According to Vieta's theorem and Liouville's formula, see Nayfeh and Mook (1979), and Yakubovich and Starzhinskii (1987), the last coefficient $c_{2}$ is equal to

$$
c_{2}=\rho_{1} \rho_{2}=|\rho|^{2}=\exp \left(\int_{0}^{2 \pi} \operatorname{tr}(\mathbf{G}) d t\right)=\exp (-2 \pi \beta)<1
$$

This inequality means that when small periodic excitation and damping are added to the unperturbed system the complex conjugate multipliers move inside the unit circle, and the system becomes asymptotically stable.

Therefore, the instability (parametric resonance) can take place only in the vicinity of the points

$$
\mathbf{p}_{0}: \varepsilon=0, \beta=0, \omega=k / 2, k=1,2, \ldots
$$

at which the multipliers are double $\rho_{1}=\rho_{2}=(-1)^{k}$. 
To find the instability domains we expand the monodromy matrix $\mathbf{F}$ in Taylor's series in the vicinity of the points $\mathbf{p}_{0}$ with respect to the parameters $\varepsilon, \beta$, and $\Delta \omega=\omega-k / 2$

$$
\mathbf{F}(\mathbf{p})=\mathbf{F}\left(\mathbf{p}_{0}\right)+\frac{\partial \mathbf{F}}{\partial \varepsilon} \varepsilon+\frac{\partial \mathbf{F}}{\partial \beta} \beta+\frac{\partial \mathbf{F}}{\partial \omega} \Delta \omega+\cdots
$$

According to formula (1.5) and with the use of (2.4)-(2.6) we calculate the derivatives $\partial \mathbf{F} / \partial \varepsilon, \partial \mathbf{F} / \partial \beta$, and $\partial \mathbf{F} / \partial \omega$ at $\mathbf{p}=\mathbf{p}_{0}$. Then, up to the first order terms (2.11) we get

$$
\mathbf{F}(\mathbf{p})=\cos \pi k\left[\begin{array}{cc}
1+\pi a_{k} \varepsilon / k-\pi \beta & 2 \pi\left(2 \Delta \omega k-b_{k} \varepsilon\right) / k^{2} \\
-\pi\left(\Delta \omega k+b_{k} \varepsilon / 2\right) & 1-\pi a_{k} \varepsilon / k-\pi \beta
\end{array}\right]
$$

Here we have introduced notation for the Fourier coefficients of the periodic function $\varphi(t)$

$$
a_{k}=\frac{1}{\pi} \int_{0}^{2 \pi} \varphi(t) \sin k t d t, \quad b_{k}=\frac{1}{\pi} \int_{0}^{2 \pi} \varphi(t) \cos k t d t, \quad k=1,2, \ldots
$$

The eigenvalues of matrix (2.12) (the multipliers) can be found approximately as

$$
\begin{gathered}
\rho_{1,2}=(-1)^{k}(1-\pi \beta) \pm \pi \sqrt{D} \\
D=r_{k}^{2} \varepsilon^{2} / k^{2}-4(\Delta \omega)^{2}, \quad r_{k}=\sqrt{a_{k}^{2}+b_{k}^{2}}
\end{gathered}
$$

The system is unstable if the absolute value of at least one multiplier is greater than one. This condition is fulfilled for $\beta<0$, and the system becomes unstable. But if $\beta \geq 0$ this instability condition is satisfied only when $\sqrt{D}>\beta$. Hence, using (2.15) we obtain the instability (parametric resonance) domain as

$$
4(\omega-k / 2)^{2}+\beta^{2}<r_{k}^{2} \varepsilon^{2} / k^{2}, \beta \geq 0
$$

It is half of the cone in three-parameter space joining the half-space $\beta<0$, Fig 1 .

Putting in (2.16) $\beta=0$ we obtain the domains of parametric resonance with no damping

$$
-\varepsilon r_{k} /(2 k)<\omega-k / 2<\varepsilon r_{k} /(2 k)
$$

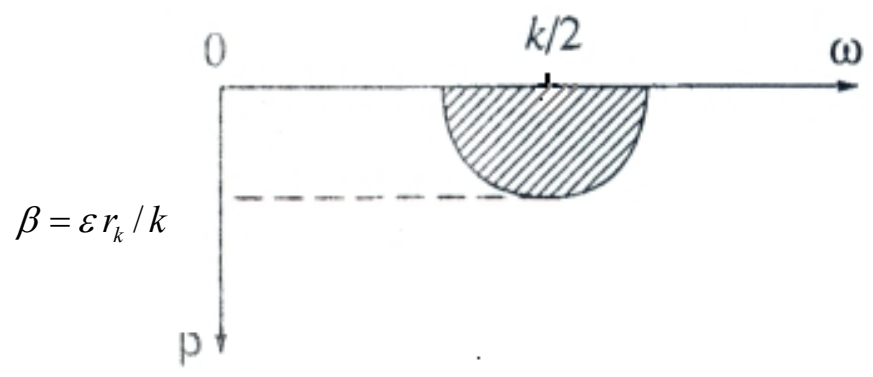

Figure 2: Cross section of the resonance domain by the plane $\varepsilon=$ const.

The cross section of the domain (2.16) by the plane $\varepsilon=$ const is half of the ellipse with the semi-axes $|\omega-k / 2|=r_{k} \varepsilon /(2 k)$ and $\beta=r_{k} \varepsilon / k$, see Fig. 2. Note that with an increase of the damping coefficient $\beta$ the width of the resonance range by the frequency $\omega$ decreases, and for $\beta>r_{k} \varepsilon / k$ disappears at all. 


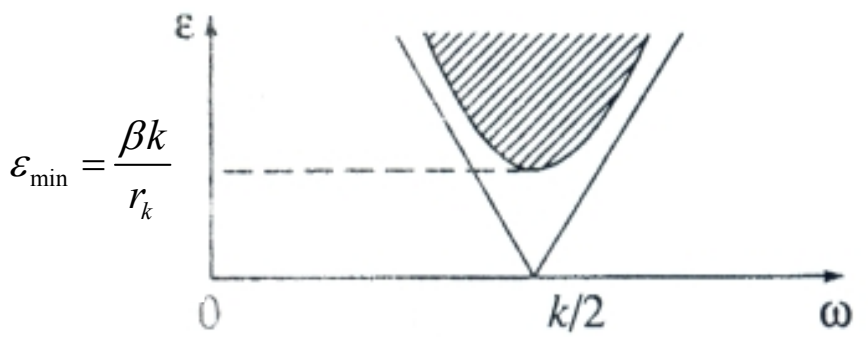

Figure 3: Cross section of the resonance domain by the plane $\beta=$ const, $\beta \geq 0$.

The cross section of the half-cone (2.16) by the plane $\beta=$ const, $\beta \geq 0$ gives the domain of parametric resonance limited by hyperbola, see Fig 3. The asymptotes of the hyperbola are found from inequalities (2.17). When damping is included $(\beta>0)$ the minimal by absolute value excitation amplitude of the resonance according to (2.16) is $\varepsilon_{\min }=\beta k / r_{k}$, Fig 3 . With an increase of the resonance number $k$ the coefficients $r_{k} / k$ tend to zero. This means that when $k$ is increasing the values $\varepsilon_{\min }$ tend to infinity and the cone is narrowing.

Formula (2.16) agrees with those obtained earlier for some specific cases. For instance, if we put in (2.16) $\beta=0, \varphi(t)=\cos t$ we get the Mathieu equation. In this case for the domain of the first resonance $k=1$ we get according to (2.13) $a_{1}=0, b_{1}=1, r_{1}=1$, and from (2.16) we obtain the well known relation $1-\varepsilon<2 \omega<1+\varepsilon$, see Nayfeh and Mook (1979) and Merkin (1997).

More general case when mean value of the periodic function is not zero was treated in Seyranian (2001) and Seyranian and Mailybaev (2003). The presented method of analysis of the parametric resonance domains using derivatives of the monodromy matrix with respect to parameters seems to be more simple and evident in comparison with the methods based on the search of periodic solutions on the boundaries of the stability domains, see Nayfeh and Mook (1979) and Merkin (1997). Besides, it readily gives the resonance domains, not only the boundaries.

4. Instability regions for a system with periodically varying moment of inertia

We consider oscillations of a vertical massless elastic shaft with a rigid disk mounted on it, Fig 4. It is assumed that there are two masses $m$ symmetrically placed on the disk and moving along radius of the disk according to the periodic law

$$
r=r_{0}+a \varphi(\Omega t), \quad \int_{0}^{2 \pi} \varphi(\tau) d \tau=0
$$

where $r_{0}$ is the initial distance of the masses from the vertical axis of the shaft, $a$ and $\Omega$ are the excitation amplitude and frequency, respectively, and $\varphi(\tau)$ is a smooth periodic function with the period $2 \pi$ with zero mean value.

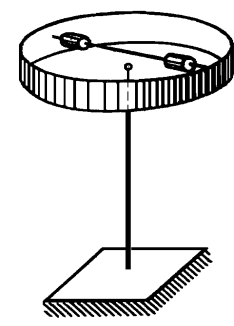

Figure 4: Elastic shaft with a rigid disk with two moving masses. 
Then the moment of inertia of the system (the disk with two masses) is equal to

$$
J(t)=J_{0}+2 m\left[r_{0}+a \varphi(\Omega t)\right]^{2}
$$

with $J_{0}$ being the moment of inertia of the disk. The equation governing free torsional oscillations of the system is

$$
(J(t) \dot{\theta})+\gamma \dot{\theta}+c \theta=0
$$

where $\theta$ is the twisting angle, $c$ is the stiffness of the shaft, and the point stands for derivative with respect to time $t$. The amplitude $a$ and the damping coefficient $\gamma$ are assumed to be small. The problem is to find regions of the parameters at which the trivial state $\theta=0$ is unstable. This classical problem without damping and with the harmonic excitation function for the first time was formulated in Panovko and Gubanova (1987).

Let us introduce non-dimensional variables and parameters

$$
\begin{gathered}
\tau=\Omega t, \varepsilon=\frac{a}{r_{0}}, \quad \varsigma=\frac{2 m r_{0}^{2}}{\tilde{J}_{0}}, \quad \tilde{J}_{0}=J_{0}+2 m r_{0}^{2}, \\
\beta=\frac{\gamma}{\sqrt{\widetilde{J}_{0} c}}, \quad \omega=\frac{1}{\Omega} \sqrt{\frac{c}{\widetilde{J}_{0}}}, \quad x_{1}=\theta, \quad x_{2}=\frac{\tilde{J}(t) \dot{\theta}}{\Omega}, \quad \widetilde{J}(t)=\frac{J(t)}{\widetilde{J}_{0}}
\end{gathered}
$$

Then equation (3.3) can be written in a form of first order equations as

$$
\frac{d x_{1}}{d \tau}=\frac{1}{\widetilde{J}(\tau)} x_{2}, \quad \frac{d x_{2}}{d \tau}=-\omega^{2} x_{1}-\frac{\beta \omega}{\widetilde{J}(\tau)} x_{2}
$$

with

$$
\widetilde{J}(\tau)=1+2 \varepsilon \varsigma \varphi(\tau)+\varepsilon^{2} \varsigma \varphi^{2}(\tau)
$$

In new variables the assumption of smoothness of the periodic function $\varphi(\tau)$ can be weakened, and it can be regarded as only piecewise continuous.

The right hand sides of equations (3.5) are linear functions of the vector $\mathbf{x}=\left(x_{1}, x_{2}\right)$ and periodic in $\tau$ with period $2 \pi$. Equations (3.5), (3.6) depend explicitly on four independent parameters $\varsigma, \omega, \varepsilon, \beta$, two last of them being small

$$
0<\omega, \quad 0<\varsigma<1, \quad 0 \leq \varepsilon<<1, \quad 0 \leq \beta<<1
$$

The problem is to find the instability (parametric resonance) regions of the trivial solution $\mathbf{x}=0 \quad$ in three-parameter space $\mathbf{p}=(\omega, \varepsilon, \beta)$ assuming the parameter $\zeta$ is fixed.

We rewrite system (3.5) in the form (1.1) with the matrix

$$
\mathbf{G}=\left\|\begin{array}{cc}
0 & {\left[1+2 \varepsilon \varsigma \varphi(\tau)+\varepsilon^{2} \varsigma \varphi^{2}(\tau)\right]^{-1}} \\
-\omega^{2} & -\beta \omega\left[1+2 \varepsilon \varsigma \varphi(\tau)+\varepsilon^{2} \varsigma \varphi^{2}(\tau)\right]^{-1}
\end{array}\right\|
$$

explicitly depending on four parameters and the periodic function $\varphi(\tau)$. We are interested in studying behavior of multipliers near the point $\mathbf{p}_{0}=(\omega, 0,0)$ and arbitrary value $\varsigma \in(0,1)$.

So, we substitute $\varepsilon=0, \beta=0$ in (1.1),(3.8) and find the matriciant and the inverse matrix from (1.2) as 


$$
\mathbf{X}_{0}(t)=\left\|\begin{array}{cc}
\cos \omega t & \omega^{-1} \sin \omega t \\
-\omega \sin \omega t & \cos \omega t
\end{array}\right\|, \quad \mathbf{X}_{0}^{-1}(t)=\left\|\begin{array}{cc}
\cos \omega t & -\omega^{-1} \sin \omega t \\
\omega \sin \omega t & \cos \omega t
\end{array}\right\|
$$

Thus, for $\varepsilon=0, \beta=0$ we have the monodromy matrix in the form

$$
\mathbf{F}_{0}=\mathbf{X}_{0}(2 \pi)
$$

The eigenvalues of this matrix (the multipliers) are

$$
\rho_{1,2}=\cos 2 \pi \omega \pm i \sin 2 \pi \omega
$$

For $\omega \neq k / 2, k=1,2, \ldots$ the multipliers are complex conjugate quantities lying on the unit circle (stability). For small changes of the parameters $\omega, \varepsilon, \beta$ in the vicinity of the point $\mathbf{p}_{0}=(\omega, 0,0), \quad \omega \neq k / 2(k=1,2, \ldots)$ the multipliers due to continuity remain complex conjugate quantities. For the multipliers we have a quadratic equation

$$
\rho^{2}+A \rho+B=0
$$

where the last term according to Liouville formula and expression (3.8) is given by

$$
B=\exp \left(\int_{0}^{2 \pi} \operatorname{tr} \mathbf{G} d t\right)=\exp \left(-2 \pi \beta \omega\left(1+o\left(\varepsilon^{2}\right)\right)\right)
$$

Due to Vieta theorem from equations (3.7), (3.13) for $\beta>0$ and sufficiently small $\varepsilon$ we have

$$
\rho_{1} \rho_{2}=B<1
$$

Then for complex conjugate multipliers from (3.14) the inequality $\left|\rho_{1,2}\right|<1$ is valid. This means that any small change of the parameters $\omega, \beta, \varepsilon$ with $\beta>0$ in the vicinity of the point $\mathbf{p}_{0}=(\omega, 0,0), \omega \neq k / 2$ shifts the multipliers inside the unit circle which guarantees asymptotic stability.

Therefore, instability (parametric resonance) can take place only in the vicinity of the points

$$
\mathbf{p}_{0}: \varepsilon=0, \beta=0, \omega=k / 2, k=1,2, \ldots
$$

at which the multipliers are double.

For finding domains of parametric resonance we expand the monodromy matrix $\mathbf{F}$ in Taylor's series in the vicinity of the points $\mathbf{p}_{0}$ with respect to the parameters $\varepsilon, \beta$, and $\Delta \omega=\omega-k / 2$

$$
\mathbf{F}(\mathbf{p})=\mathbf{F}\left(\mathbf{p}_{\mathbf{0}}\right)+\frac{\partial \mathbf{F}}{\partial \varepsilon} \varepsilon+\frac{\partial \mathbf{F}}{\partial \beta} \beta+\frac{\partial \mathbf{F}}{\partial \omega} \Delta \omega+\cdots
$$

According to formulas (1.5) and using (3.8)-(3.10) we calculate the derivatives $\partial \mathbf{F} / \partial \varepsilon, \partial \mathbf{F} / \partial \beta$, and $\partial \mathbf{F} / \partial \omega$ at $\mathbf{p}=\mathbf{p}_{\mathbf{0}}$. Then, up to first order accuracy (3.16) we get

$$
\mathbf{F}(\mathbf{p})=\cos \pi k\left\|\begin{array}{cc}
1+\left(\pi b_{k} k \varepsilon \varsigma-\pi \beta k\right) / 2 & 4 k^{-1} \pi \Delta \omega-\pi a_{k} \varepsilon \varsigma \\
-k \pi \Delta \omega-\pi a_{k} k^{2} \varepsilon \varsigma / 4 & 1-\left(\pi b_{k} k \varepsilon \varsigma+\pi \beta k\right) / 2
\end{array}\right\|
$$

Here, the quantities $a_{k}, b_{k}, k=1,2, \ldots$ are Fourier coefficients of the periodic function $\varphi(\tau)$.

The eigenvalues of the matrix (3.17) (the multipliers) can be found approximately as

$$
\rho_{1,2}=(-1)^{k}(1-\pi \beta k / 2) \pm \pi \sqrt{D}
$$




$$
D=r_{k}^{2} k^{2} \varepsilon^{2} \varsigma^{2} / 4-(2 \Delta \omega)^{2}, r_{k}=\sqrt{a_{k}^{2}+b_{k}^{2}}
$$

The system is unstable if the absolute value of at least one multiplier is greater than one. This condition is fulfilled for $\beta<0$, and the system becomes unstable. But if $\beta \geq 0$ this condition is satisfied only when $\sqrt{D}>\beta k / 2$. Hence, using (3.18) we obtain the regions of parametric resonance as

$$
4(2 \omega / k-1)^{2}+\beta^{2}<r_{k}^{2} \varepsilon^{2} \varsigma^{2}, \beta \geq 0
$$

This is half of the cone joining the half-space $\beta<0$, Fig 1. Formula (3.19) is the first order approximation of the instability domains showing that the k-th resonance domain depends only on the k-th Fourier coefficients of the periodic excitation function. If $a_{k}=0$ and $b_{k}=0$, which implies $r_{k}=0$, then the k-th resonance domain degenerates to the straight line $\beta=0$ and $\omega=k / 2$. In this case to determine the resonance domain more accurately higher order approximations are needed. It can also mean that the corresponding resonance domain is empty.

The cross section of the half-cone (3.19) by the plane $\beta=$ const, $\beta \geq 0$ gives the zones of parametric resonance limited by hyperbolae, see Fig 3 . When damping is included $(\beta>0)$ the minimal excitation amplitude of the resonance according to (3.19) is equal to

$$
\varepsilon_{\min }=\beta /\left(r_{k} \varsigma\right)
$$

With an increase of the resonance number $k$ the Fourier coefficients $a_{k}, b_{k}$, and hence $r_{k}$, tend to zero. This means that for a fixed damping coefficient $\beta$ and parameter $\zeta$ with an increase of $k$ the minimal excitation amplitude tends to infinity. This explains why it is easier to observe the parametric resonance at small numbers $k=1,2, \ldots$ because at higher resonance numbers essential efforts and high excitation amplitudes are needed to set the system into unstable motion.

From expression (3.19) and presented analysis it is easy to see that with increasing parameter $\varsigma, 0<\varsigma<1$, resonance domains get wider. According to (3.4) this means that when the moment of inertia of the masses increases, keeping fixed the moment of inertia of the disk, then the resonance domains become wider.

Using (3.4), (3.15) we find that the parametric resonance takes place near the critical frequencies

$$
\Omega_{c r}=\frac{2 \Omega_{0}}{k}, \quad k=1,2, \ldots ; \quad \Omega_{0}=\sqrt{\frac{c}{J_{0}+2 m r_{0}^{2}}}
$$

We note that $\Omega_{0}$ is the eigenfrequency of torsional oscillations of the disk with two immovable masses $m$ taken at the distance $r_{0}$ from the rotation axis. With the use of (3.4) and (3.15) resonance domains (3.19) can be given in dimensional form.

As a numerical example we take $\varphi(\tau)=\cos \tau$ and $\varsigma=1 / 2$. In this case for the first resonance domain $k=1$ we get from (2.13) $a_{1}=r_{1}=1, b_{1}=0$. Thus, according to (3.4) and (3.19) we obtain the explicit expression for the first resonance domain in dimensional form as

$$
4\left(\frac{\Omega}{2 \Omega_{0}}-1\right)^{2}+\frac{\gamma^{2}}{\left(J_{0}+2 m r_{0}^{2}\right) c}<\frac{a^{2}}{4 r_{0}^{2}}
$$

We note that all the other resonance regions are degenerate since $a_{k}=b_{k}=r_{k}=0, \quad k=2,3,4 \ldots$

As another example we take a continuously differentiable $2 \pi$ - periodic function 


$$
\varphi(\tau)=\left\{\begin{aligned}
\tau(\pi-\tau), & 0 \leq \tau \leq \pi \\
(\pi-\tau)(2 \pi-\tau), & \pi<\tau \leq 2 \pi
\end{aligned}\right.
$$

consisting of two parabolas with positive and negative curvature. For odd Fourier coefficients of this function we have

$$
\begin{aligned}
& a_{k}=0, b_{k}=r_{k}=\frac{8}{\pi k^{3}}, k=1,3,5, \ldots ; \\
& a_{k}=b_{k}=r_{k}=0, \quad k=2,4,6, \ldots
\end{aligned}
$$

Thus, formula (3.19) for odd resonance domains yields

$$
4\left(\frac{2 \omega}{k}-1\right)^{2}+\beta^{2}<\frac{64 \varepsilon^{2} \varsigma^{2}}{\pi^{2} k^{6}}, k=1,3,5 \ldots
$$

showing how fast with growth of $k$ the resonance regions shrink, while even resonance domains are degenerate.

\section{Stabilization of an inverted pendulum}

Vibration and stability of an inverted pendulum were studied by many authors. Among classical works we cite Stephenson (1908) and Kapitsa (1951). The difference between the present paper and previous research is that we assume existence of viscous damping forces, consider arbitrary periodic excitation function, and use the method of stability study of periodic systems based on analysis of the Floquet multipliers.

Oscillations of a physical pendulum with the vibrating suspension point about upper vertical (statically unstable) position is described by the equation

$$
I \theta_{t t}+c \theta_{t}-m r\left(g+z_{t t}\right) \sin \theta=0
$$

Here $I$ and $m$ are the moment of inertia and mass of the pendulum, $\theta$ is the angle measured from the vertical axis, $C$ is the damping coefficient, $r$ is the distance between suspension point and the center of gravity, $g$ is the acceleration due to gravity, $z$ is the vertical displacement of the suspension point following the law $z=a \phi(\Omega t)$, where $\Omega$ is the excitation frequency, and $\phi(\tau)$ is an arbitrary $2 \pi$-periodic function. The amplitude $a$ and damping coefficient $c$ are supposed to be small. For the sake of simplicity we use notation $\varphi=-\phi_{t t}$ and assume that the mean value of the periodic function $\varphi(\tau)$ is equal to zero.

We introduce non-dimensional time and parameters

$$
\tau=\Omega t, \quad \beta=\frac{c}{I \Omega}, \quad \varepsilon=\frac{a \Omega_{0}^{2}}{g}, \quad \omega=\frac{\Omega_{0}}{\Omega}, \quad \Omega_{0}=\sqrt{\frac{m r g}{I}}
$$

where $\Omega_{0}$ is the eigenfrequency of the pendulum with fixed suspension point about lower vertical position.

With this notation linearized equation (4.1) takes the form of Hill's equation with damping

$$
\ddot{\theta}+\beta \dot{\theta}+[\mu+\varepsilon \varphi(\tau)] \theta=0, \quad \mu=-\omega^{2}
$$

where the dot denotes differentiation with respect to $\tau$. The coefficients of this equation explicitly depend on the $2 \pi$-periodic function $\varphi(\tau)$ and three non-dimensional parameters $\varepsilon, \beta, \omega$. The amplitude and damping parameters $\varepsilon$ and $\beta$ are assumed to be small with respect to 1 .

At $\varepsilon=0, \beta=0$ the upper vertical position of the pendulum $\theta=0$ is unstable. Let us study possibility of stabilization of this position by high-frequency excitation of the 
suspension point. In this case we are close to the point $\varepsilon=0, \beta=0, \mu=0$. To find the stability region in the vicinity of this point we use the method based on calculation of derivatives of the monodromy matrix and analysis of Floquet multipliers. We show that the stability region for an arbitrary periodic function $\varphi(\tau)$ in the vicinity of the point $\mathbf{p}_{0}=(0,0,0)$ in the first approximation is given by the inequality

$$
\mu>F \varepsilon^{2}, \quad F=\left(\frac{1}{2 \pi} \int_{0}^{2 \pi} t \varphi(t) d t\right)^{2}-\frac{1}{\pi} \int_{0}^{2 \pi} \varphi(t) \int_{0}^{t} \tau \varphi(\tau) d \tau d t
$$

It can be shown that for most of the periodic functions the constant $F<0$. In the formula for stabilization region the terms of higher order have the form of rather complicated multiple integrals.

For the function $\varphi(\tau)=\cos \tau$ we obtain the stabilization region near the point $\mathbf{p}_{0}=(0,0,0)$ as

$$
\mu>-\frac{\varepsilon^{2}}{2}+\frac{\varepsilon^{2} \beta^{2}}{2}+\frac{7 \varepsilon^{4}}{32}
$$

From this formula we can see that damping has destabilizing effect on the stability region. Influence of damping to the stability boundary is shown in Fig. 5 for the case of the periodic function $\varphi(\tau)=\cos \tau$. The analytical results with (4.5) are shown by solid line, and the numerical results are shaded. Fig. 5 shows that analytical and numerical results are in a good agreement with each other up to the values $\varepsilon \approx 0.5, \beta \approx 0.4, \mu \approx-0.15$.

Using formulas for stability regions for Hill's equation with damping we can obtain the stabilization frequency of the pendulum. With introduction of the damping coefficient as $\beta_{0}=c /\left(I \Omega_{0}\right)$ for the function $\varphi(\tau)=\cos \tau$ we obtain a formula for the stabilization frequency in the form

$$
\frac{\Omega}{\Omega_{0}}>\sqrt{2}\left[\frac{1}{\varepsilon}+\frac{7 \varepsilon}{32}+\frac{\varepsilon \beta_{0}^{2}}{4}-\frac{2389 \varepsilon^{3}}{18432}\right]
$$

Thus, at rather high excitation frequency the upper vertical position of the pendulum becomes stable, and small damping increases the critical stabilization frequency. The first term in the right hand side of (4.6) agrees with that of known in the literature, see e.g. Kapitsa (1951).

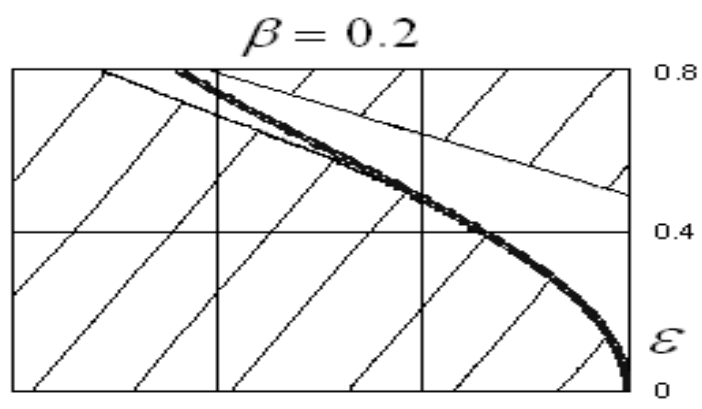




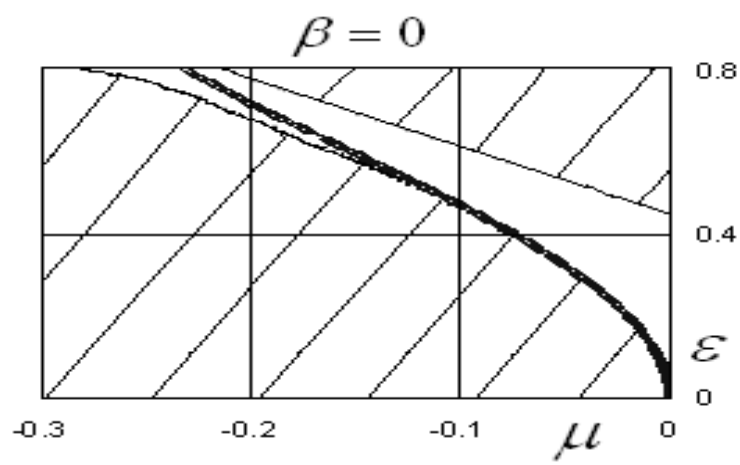

Fig. 5: Stability region for the Hill equation with damping

If we consider a mathematical pendulum with piecewise constant excitation function

$$
\varphi(\tau)=\left\{\begin{aligned}
1, & 0<\tau \leq \pi \\
-1, & \pi<\tau<2 \pi
\end{aligned}\right.
$$

for which we have $F=-\pi^{2} / 12$, then we obtain the stabilization condition as

$$
\frac{\Omega}{\Omega_{0}}>\sqrt{3}\left[\frac{2}{\pi \varepsilon}+\frac{\pi^{3} \varepsilon}{126}+\frac{\pi^{3} \varepsilon \beta_{0}^{2}}{120}-\frac{79 \pi^{7} \varepsilon^{3}}{970200}\right]
$$

The formula with only first term in the right hand side of (4.8) was derived in Arnold (1978). However, the critical stabilization frequency found in Arnold (1978) is in $\sqrt{2}$ higher than it should be.

For the periodic function with no symmetry $\varphi(\tau)=2(\tau / 2 \pi)^{3}-1 / 2,0 \leq \tau \leq 2 \pi$, the stability condition takes the form

$$
\frac{\Omega}{\Omega_{0}}>\frac{2.19}{\varepsilon}-0.202+0.162 \varepsilon+0.045 \varepsilon^{2}+0.214 \varepsilon \beta_{0}^{2}-0.028 \varepsilon^{3}
$$

We note that in this formula there are terms of zero and second order with respect to $\varepsilon$. As earlier, taking damping into account leads to increase in the critical stabilization frequency. Formulas (4.6), (4.8), (4.9) are in a good agreement with numerical results up to the values $\varepsilon \approx 0.8, \beta_{0} \approx 0.3$.

\section{Stability of periodic solutions in nonlinear systems}

In this section using the results on the instability domains for Hill's equation with damping we show how to study stability of periodic solutions in nonlinear dynamical systems. For this purpose we consider harmonically excited Duffing's equation, see Nayfeh and Mook (1979) and Thomsen (1997)

$$
\ddot{u}+2 \mu \dot{u}+\omega_{0}^{2} u+\alpha u^{3}=f \cos \Omega t
$$

Here we assume that the excitation frequency is close to the natural frequency $\Omega=\omega_{0}+\sigma$ (primary resonance), and the constants $\mu, \alpha, f$ and $\sigma$ are small quantities of the same order $o(1)$. Equation (5.1) admits an approximate periodic solution, see Thomsen (1997)

$$
u_{0}(t)=a \cos (\Omega t-\gamma)+\frac{\alpha a^{3}}{32 \omega_{0}^{2}} \cos (3 \Omega t-3 \gamma)
$$

where the amplitude $a$ and phase $\gamma$ are constants of the order $O(1)$ satisfying the equations 


$$
\begin{gathered}
{\left[\left(\Omega-\omega_{0}-\frac{3 \alpha a^{2}}{8 \omega_{0}}\right)^{2}+\mu^{2}\right] a^{2}=\frac{f^{2}}{4 \omega_{0}^{2}}} \\
\gamma=\arcsin \left(\frac{2 \mu a \omega_{0}}{f}\right)
\end{gathered}
$$

For given constants $\Omega, \omega_{0}, \alpha, \mu, f$ (5.3) is a cubic equation on the unknown squared amplitude $a^{2}$. The plot of $a$ as a function of $\Omega$ for given other quantities is called a frequency-response curve. To draw this curve it is convenient to express $\Omega$ from (5.3) as a function of $a$

$$
\Omega=\omega_{0}+\frac{3 \alpha a^{2}}{8 \omega_{0}} \pm \sqrt{\frac{f^{2}}{4 \omega_{0}^{2} a^{2}}-\mu^{2}}
$$

This formula gives two branches $\Omega_{+}$and $\Omega_{-}$. A typical frequency-response curve for $\alpha>0$ is presented in Fig 6 . Note that the cases $\alpha>0$ and $\alpha<0$ are symmetric with respect to the vertical axis $\Omega=\omega_{0}$. Such multivaluable behavior $a(\Omega)$ is typical for nonlinear systems. For some values $\Omega$ it is possible to have three different regimes $a$ and $\gamma$ of the periodic solution (5.2). Some of them might be stable, and some unstable.

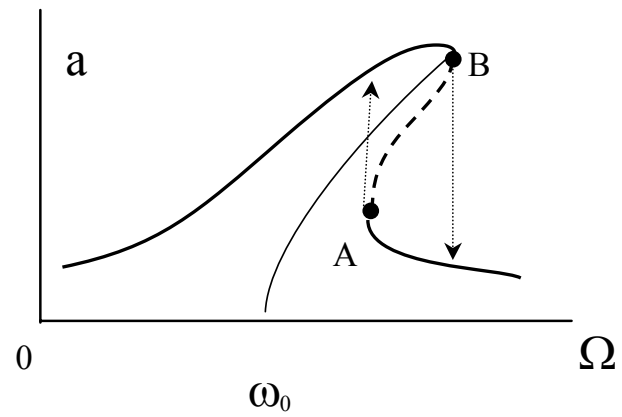

Figure 6: The frequency-response curve for Duffing's equation.

To study the stability of the periodic solution (5.2) we take

$$
u(t)=u_{0}(t)+v(t)
$$

substitute this expression into (5.1), and then linearize with respect to $v(t)$. Using (5.2) and (5.6) we get, see Nayfeh and Mook (1979)

$$
\ddot{v}+2 \mu \dot{v}+\left[\omega_{0}^{2}+3 \alpha a^{2} \cos ^{2}(\Omega t-\gamma)\right] v=0
$$

The stability of the periodic solution (5.2) is governed by equation (5.7). If the solution $v(t)$ to this equation is stable, the periodic motion (5.2) is stable, and vice versa. Introducing the notation $\tau=\Omega t-\gamma$ we obtain in the first approximation

$$
\frac{d^{2} v}{d \tau^{2}}+\frac{2 \mu}{\omega_{0}} \frac{d v}{d \tau}+\left[1-\frac{2 \sigma}{\omega_{0}}+\frac{3 \alpha a^{2}}{\omega_{0}^{2}} \cos ^{2} \tau\right] v=0
$$

Using here $\cos ^{2} \tau=(1+\cos 2 \tau) / 2$ we get Hill's equation in the form 


$$
\frac{d^{2} v}{d \tau^{2}}+\frac{2 \mu}{\omega_{0}} \frac{d v}{d \tau}+\left[1-\frac{2 \sigma}{\omega_{0}}+\frac{3 \alpha a^{2}}{2 \omega_{0}^{2}}+\frac{3 \alpha a^{2}}{2 \omega_{0}^{2}} \cos 2 \tau\right] v=0
$$

Comparison with equation (2.1) implies

$$
\beta=\frac{2 \mu}{\omega_{0}}, \quad \omega^{2}=1-\frac{2 \sigma}{\omega_{0}}+\frac{3 \alpha a^{2}}{2 \omega_{0}^{2}}, \quad \varepsilon=\frac{3 \alpha a^{2}}{2 \omega_{0}^{2}}, \quad \varphi(\tau)=\cos 2 \tau
$$

Calculating the coefficients according to (2.13) and (2.15) we find

$$
k=2, \quad a_{2}=0, \quad b_{2}=1, \quad r_{2}=1
$$

Then the instability condition (2.16) yields the cone

$$
\left(\Omega-\omega_{0}-\frac{3 \alpha a^{2}}{4 \omega_{0}}\right)^{2}+\mu^{2}<\left(\frac{3 \alpha a^{2}}{8 \omega_{0}}\right)^{2}
$$

However, unlike the linear case the excitation frequency $\Omega$ and the amplitude $a$ are not independent quantities due to relations (5.5). Condition (5.12) can be transformed to

$$
\left(\Omega-\omega_{0}-\frac{3 \alpha a^{2}}{8 \omega_{0}}\right)\left(\Omega-\omega_{0}-\frac{9 \alpha a^{2}}{8 \omega_{0}}\right)+\mu^{2}<0
$$

In this form the instability condition was derived in Nayfeh and Mook (1979) and Thomsen (1997) using the method of multiple scales. Verifying condition (5.13) for the branch $\Omega_{-}$from (5.5) for $\alpha>0$ we obtain the inequality of the opposite sign implying the stability of the periodic solution (5.2). Substituting in (5.13) the branch $\Omega_{+}$from (5.5) we obtain that for $\alpha>0$ it is satisfied only when

$$
a_{*}<a<a_{* *}
$$

where $a_{*}$ and $a_{* *}$ are the two roots of the equation

$$
\frac{f^{4}}{9 \alpha^{2} \omega_{0}^{2} a^{8}}=\frac{f^{2}}{4 \omega_{0}^{2} a^{2}}-\mu^{2}
$$

We note that equation (5.15) is equivalent to the condition $d \Omega_{+} / d a=0$ indicating that the unstable regime (5.2) lies between the points A and B shown in Fig. 6 by dashed line. Other parts of the branch $\Omega_{+}$, shown in Fig. 6 by solid line, correspond to the stable periodic solutions (5.2). The points $\mathrm{A}$ and $\mathrm{B}$ are the catastrophe points due to a jump in the amplitude $a$. We emphasize that for $\omega_{0}>0, \mu>0, \alpha>0, f \neq 0$ these two points are always existing since equation (5.15) possesses the two real roots $a_{*}<a_{* *}$. The case $\alpha<0$ can be treated similarly.

Another example of stability study of a limit cycle in periodic systems was investigated both theoretically and experimentally in Seyranian, Yabuno and Tsumoto (2005). 


\section{The general case of systems with multiple degrees of freedom}

In this section we briefly describe the general case following the papers by Seyranian and Mailybaev (2001), Mailybaev and Seyranian (2001), and Seyranian and Mailybaev (2003). This case is much more complicated compared with that of for one-degree-of-freedom. However, for some important cases considered below the results for parametric resonance domains seem to be similar.

We consider a linear oscillatory system with periodic coefficients

$$
\mathbf{M} \ddot{\mathbf{y}}+\gamma \mathbf{D} \dot{\mathbf{y}}+[\mathbf{C}+\delta \mathbf{B}(\Omega t)] \mathbf{y}=\mathbf{0}
$$

Here, $\mathbf{M}, \mathbf{D}$, and $\mathbf{C}$ are the symmetric positive-definite square $m \times m$ matrices of mass, damping, and potential forces, respectively; $\mathbf{B}(\tau)$ is a piecewise continuous $2 \pi$-periodic matrix of a parametric excitation; $\mathbf{y}$ is the vector of the generalized coordinates.

We analyze the stability of the trivial solution to system (6.1) as a function of the vector of three parameters $\mathbf{p}=(\gamma, \delta, \Omega)$ whose components describe the magnitude of dissipative forces, and the amplitude and frequency of the periodic excitation, respectively.

We assume that $0<\gamma,|\delta|<<1$, i.e. system (6.1) is close to the autonomous conservative system

$$
\mathbf{C u}=\omega^{2} \mathbf{M u}, \quad \mathbf{u}^{\mathbf{T}} \mathbf{M u}=1
$$

with the simple eigenfrequencies $0<\omega_{1}<\omega_{2}<\cdots<\omega_{m}$ and the corresponding eigenmodes $\mathbf{u}_{\mathbf{1}}, \mathbf{u}_{\mathbf{2}}, \cdots, \mathbf{u}_{m}$.

We rewrite (6.1) as a set of equations of the first order:

$$
\dot{\mathbf{x}}=\mathbf{A}(\Omega t) \mathbf{x}, \quad \mathbf{x}=\left(\begin{array}{c}
\mathbf{y} \\
\dot{\mathbf{y}}
\end{array}\right)
$$

The $2 \mathrm{~m} \times 2 \mathrm{~m}$ matrix $\mathbf{A}(\Omega \mathrm{t})$ is a real-valued periodic function with the period $\mathrm{T}=2 \pi / \Omega$. The $2 \mathrm{~m} \times 2 \mathrm{~m}$ matrix $\mathbf{X}(t)$ satisfying the relationships

$$
\dot{\mathbf{X}}=\mathbf{A}(\Omega t) \mathbf{X}, \quad \mathbf{X}(0)=\mathbf{I}
$$

is referred to as a matriciant of system (6.3), with $\mathbf{I}$ being the unit matrix. The value of the matriciant at $t=T$ is referred to as a monodromy matrix $\mathbf{F}=\mathbf{X}(T)$, see Yakubovich and Starzhinski (1987). According to the known theorem on the parameter dependence of solutions to differential equations, the monodromy matrix is a smooth function of the parameter vector $\mathbf{p}$. The eigenvalues $\rho$ of the matrix $\mathbf{F}$ are called multipliers. System (6.1) (or (6.3)) is asymptotically stable if all its multipliers are located inside the unit circle on the complex plane, i.e. $|\rho|<1$. If at least one multiplier is outside of the unit circle, i.e., $|\rho|>1$, then the system becomes unstable, see Yakubovich and Starzhinski (1987).

When $\gamma=\delta=0$, the multipliers of the monodromy matrix $\mathbf{F}_{0}$ are given by, see Seyranian and Mailybaev (2003)

$$
\rho_{j}, \bar{\rho}_{j}=\exp \left( \pm i \omega_{j} T\right)=\exp \left( \pm \frac{2 \pi i \omega_{j}}{\Omega}\right) \quad, \quad j=1, \cdots, m
$$

Since all the multipliers $\rho_{j}$ from (6.5) lie on the unit circle $\left|\rho_{j}\right|=1$, the stability of system (6.1) for nonzero $\gamma$ and $\delta$ is determined by perturbations of all the multipliers. In general, all $\rho_{j}$ are different. Repeated (multiple) multipliers appear for the following critical values of the frequency $\Omega$

$$
\Omega=\frac{2 \omega_{j}}{k}, \quad j=1, \ldots, m, \quad k=1,2, \ldots
$$




$$
\Omega=\frac{\omega_{j} \pm \omega_{l}}{k}, \quad j, l=1, \ldots, m, \quad j>l ; \quad k=1,2, \ldots
$$

Equalities (6.6) and (6.7) define, respectively, the main (simple) and combination resonances of summed and difference types, and correspond to the double multipliers $\rho=(-1)^{k}$ and $\rho=\exp \left(i \omega_{j} T\right)$. Multipliers of higher order multiplicity appear only if the quantities $\omega_{j} \pm \omega_{l}$ are linked by rational relationships. These cases are nongeneric and will not be considered in this paper.

Two important cases of the symmetric excitation matrix $\mathbf{B}(\tau)=\mathbf{B}^{\mathbf{T}}(\tau)$ and the excitation matrix in the form $\mathbf{B}(\tau)=\mathbf{B}_{0} \varphi(\tau)$, where $\mathbf{B}_{0}$ is a constant matrix, and $\varphi(\tau)$ is a scalar $2 \pi$ periodic function, are considered. The parametric resonance domains for these two cases were derived in the form of half-cones, see Seyranian and Mailybaev (2003):

$$
\eta_{j} \eta_{l} \gamma^{2} \mp \xi_{1} \delta^{2}+4 k^{2} \frac{\eta_{j} \eta_{l}}{\left(\eta_{j}+\eta_{l}\right)^{2}}\left(\Delta \Omega+\frac{\sigma_{ \pm} \delta}{k}\right)^{2} \leq 0
$$

with the coefficients

$$
\begin{gathered}
\eta_{j}=\mathbf{u}_{j}^{\mathbf{T}} \mathbf{D} \mathbf{u}_{j}, \quad \eta_{l}=\mathbf{u}_{l}^{\mathbf{T}} \mathbf{D} \mathbf{u}_{l}, \quad \xi_{1}=\frac{a_{k}^{j l} a_{k}^{l j}+b_{k}^{j l} b_{k}^{l j}}{4 \omega_{j} \omega_{l}} \\
a_{k}^{j l}=\frac{1}{\pi} \int_{0}^{2 \pi} \mathbf{u}_{j}^{\mathbf{T}} \mathbf{B}(\tau) \mathbf{u}_{l} \cos (k \tau) d \tau, \quad b_{k}^{j l}=\frac{1}{\pi} \int_{0}^{2 \pi} \mathbf{u}_{j}^{\mathbf{T}} \mathbf{B}(\tau) \mathbf{u}_{l} \sin (k \tau) d \tau \\
\Delta \Omega=\Omega-\frac{\omega_{j} \pm \omega_{l}}{k}, \quad \sigma_{ \pm}=-\frac{\omega_{j} a_{0}^{j j} \pm \omega_{l} a_{0}^{l l}}{4 \omega_{j} \omega_{l}}
\end{gathered}
$$

Plus and minus in (6.8),(6.9) correspond to the combination resonances (6.7) of summed or difference types, respectively. For the main resonance (6.6) we have to put in (6.8), (6.9) $j=l$. For $\xi_{1} \neq 0$ (the regular case), only one of the inequalities (6.8) with plus or minus defines a cone, while the other one gives the point $\gamma=\delta=\Delta \Omega=0$ (absence of resonance). Therefore, for positive $\xi_{1}$ the domain of the difference-type combination resonance is lacking.

Consider now the case of the symmetric excitation matrix $\mathbf{B}(\tau)=\mathbf{B}^{\mathbf{T}}(\tau)$. It is easy to notice from (6.9) that in this case $a_{k}^{j l}=a_{k}^{l j}$ and $b_{k}^{j l}=b_{k}^{l j}$ which means $\xi_{1}>0$. Thus, for the symmetric excitation matrix only the main and combination resonances of summed type take place. It is worth noting that, in the case of Hamiltonian systems (without dissipation), the absence of the difference-type combination resonances has already been noted, see Yakubovich and Starzhinski (1987).

Consider now the case of the excitation matrix $\mathbf{B}(\tau)=\mathbf{B}_{0} \varphi(\tau)$, where $\mathbf{B}_{0}$ is a constant matrix, and $\varphi(\tau)$ is a scalar $2 \pi$ periodic function. In this case we have from (6.9)

$$
\begin{gathered}
\xi_{1}=\frac{c_{j l}\left(a_{k}^{2}+b_{k}^{2}\right)}{4 \omega_{j} \omega_{l}}, \quad c_{j l}=\mathbf{u}_{j}^{\mathrm{T}} \mathbf{B}_{0} \mathbf{u}_{l} \mathbf{u}_{l}^{\mathrm{T}} \mathbf{B}_{0} \mathbf{u}_{j}, \\
a_{k}=\frac{1}{\pi} \int_{0}^{2 \pi} \varphi(\tau) \cos (k \tau) d \tau, \quad b_{k}=\frac{1}{\pi} \int_{0}^{2 \pi} \varphi(\tau) \sin (k \tau) d \tau
\end{gathered}
$$


The main and combination resonances are given by relations (6.8), (6.9). In the regular (nondegenerate) case, when $\xi_{1} \neq 0$, the sign of $\xi_{1}$ coincides with that of $c_{j l}$. For the main resonance, $c_{j j}>0$; therefore, there exists the main-resonance domain defined by cone (6.8). The existence of combination resonance domains depends on the sign of $c_{j l}$. Namely, for $c_{j l}>0$ or $c_{j l}<0$, only the domain of the summation-type or difference-type combination resonance exists, respectively. The shape of the parametric-resonance domains (cones) depends on the resonance number $k$ and the frequencies $\omega_{j}$ and $\omega_{l}$. When $c_{j l}=0$, the resonance domain is either absent or degenerate (i.e., the first approximation represents a straight line). We notice that with an increase of $k$ and $\omega_{j}, \omega_{l}$ the cones (6.8) become narrow and straight, Fig 1. We formulate the results obtained in the following statement.

Theorem. For a symmetric matrix $\mathbf{B}(\tau)=\mathbf{B}^{\mathbf{T}}(\tau)$ system (6.1) exhibits only the main resonances (6.6) and the summation-type combination resonances (6.7). In the case of $\mathbf{B}(\tau)=\mathbf{B}_{0} \varphi(\tau)$, where $\mathbf{B}_{0}$ is a constant matrix, and $\varphi(\tau)$ is a scalar $2 \pi$ periodic function, either the main resonances (6.6), the summation-type combination resonances (for $c_{j l}>0$ ), or the difference-type combination resonances (for $c_{j l}<0$ ) are realized in the system. In the three-dimensional space of the parameters $\mathbf{p}=(\gamma, \delta, \Omega)$, the domains of both main and summation-type combination resonances, as well as difference combination resonances are described by cones (6.8).

The cases of excitation matrices considered above correspond to the most conventional forms of parametric excitations. In other cases, the parametric resonance domains are described by much more complicated formulas, see Mailybaev and Seyranian (2001) and Seyranian and Mailybaev (2003).

\section{Bolotin's problem}

As a mechanical example, we consider a problem on dynamic stability of the trivial solution (plane position) of a beam. The elastic beam is assumed to be simply supported at its ends and loaded by the periodic moments $M(\Omega t)=\delta \varphi(\Omega t)$ in the plane of its maximum stiffness, where $\varphi(t)$ is a $2 \pi$-periodic function, Fig. 7. We note that this problem for the first time was formulated in Bolotin (1964), see also Bolotin (1995).

Bending-torsional vibrations off this plane are described by the equations

$$
\begin{aligned}
& m \frac{\partial^{2} w}{\partial t^{2}}+m d_{1} \frac{\partial w}{\partial t}+E J \frac{\partial^{4} w}{\partial x^{4}}+\delta \varphi(\Omega t) \frac{\partial^{2} \theta}{\partial x^{2}}=0 \\
& m r^{2} \frac{\partial^{2} \theta}{\partial t^{2}}+m m r^{2} d_{2} \frac{\partial \theta}{\partial t}+\delta \varphi(\Omega t) \frac{\partial^{2} w}{\partial x^{2}}-G I \frac{\partial^{2} \theta}{\partial x^{2}}=0
\end{aligned}
$$

Here, $w(x, t)$ is the transverse deflection of the beam; $\theta(x, t)$ and $r$ are the torsion angle and the radius of inertia for the beam's cross section, respectively; $E J$ and $G I$ are the bending and torsion stiffnesses of the beam, respectively; $m$ is the mass per unit length of the beam; $\gamma$ is the parameter of dissipative force (viscous friction coefficient); and $d_{1}$ and $d_{2}$ are fixed constants defining the bending and torsional viscous friction forces.

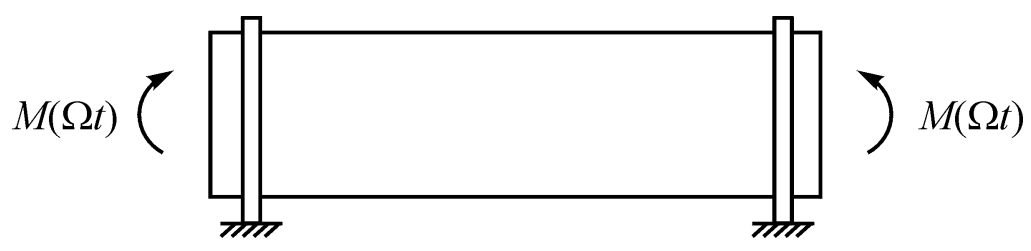

Figure 7: An elastic beam loaded by periodic bending moments. 
The boundary conditions take the form

$$
x=0, l: \quad w=\frac{\partial^{2} w}{\partial x^{2}}=\theta=0
$$

where $l$ is the beam length. We seek the solution to system (7.1), (7.2) in the form of series:

$$
\begin{aligned}
& w(x, t)=\sum_{n=1}^{\infty} W_{n}(t) \sin \frac{n \pi x}{l} \\
& \theta(x, t)=\sum_{n=1}^{\infty} \Theta_{n}(t) \sin \frac{n \pi x}{l}
\end{aligned}
$$

where $W_{n}(t)$ and $\Theta_{n}(t)$ are unknown functions of time. Substituting (7.3) into (7.1), we obtain a set of ordinary differential equations with respect to $W_{n}(t)$ and $\Theta_{n}(t)$ of the form of (6.1) with

$$
\begin{gathered}
\mathbf{M}=\left(\begin{array}{ll}
1 & 0 \\
0 & 1
\end{array}\right), \quad \mathbf{D}=\left(\begin{array}{ll}
d_{1} & 0 \\
0 & d_{2}
\end{array}\right), \quad \mathbf{C}=\left(\begin{array}{cc}
\omega_{n 1}^{2} & 0 \\
0 & \omega_{n 2}^{2}
\end{array}\right) \\
\mathbf{B}(\Omega t)=-\varphi(\Omega t)\left(\begin{array}{cc}
0 & \pi^{2} n^{2} /\left(l^{2} m\right) \\
\pi^{2} n^{2} /\left(l^{2} r^{2} m\right) & 0
\end{array}\right), \quad \mathbf{y}=\left(\begin{array}{l}
W_{n} \\
\Theta_{n}
\end{array}\right)
\end{gathered}
$$

Here, $\omega_{n 1}$ and $\omega_{n 2}$ are the natural frequencies of bending and torsional vibrations of the beam, respectively:

$$
\omega_{n 1}=\frac{n^{2} \pi^{2}}{l^{2}} \sqrt{\frac{E J}{m}}, \quad \omega_{n 2}=\frac{n \pi}{r l} \sqrt{\frac{G I}{m}}, \quad n=1,2, \cdots
$$

The eigenvectors corresponding to the frequencies $\omega_{n 1}$ and $\omega_{n 2}$ are equal to $\mathbf{u}_{\mathrm{n} 1}=(1,0)^{\mathrm{T}}$ and $\mathbf{u}_{\mathrm{n} 2}=(0,1)^{\mathrm{T}}$, respectively. We now analyze the stability of systems (6.1) with (7.4),(7.5) for a certain fixed $n$. Since $\mathbf{B}(\tau)=\mathbf{B}_{0} \varphi(\tau)$, where $\mathbf{B}_{0}$ is a constant matrix, and $\varphi(\tau)$ is a scalar $2 \pi$ periodic function, this system belongs to the second type considered above in section 6 . The quantities defined in $(6.10)$ take the form

$$
c_{11}=c_{22}=0, \quad c_{12}=\frac{\pi^{4} n^{4}}{l^{4} r^{2} m^{2}}>0
$$

Therefore, the domains of the difference-type combination resonance are absent, while the main resonance domains are degenerate (in order to analyze them, higher order approximations are required). According to (6.8), the domains of the summation-type combination resonance at frequencies close to $\Omega_{0}=\left(\omega_{n 1}+\omega_{n 2}\right) / k, k=1,2, \ldots$ are determined by

$$
d_{1} d_{2} \gamma^{2}-\frac{c_{12}\left(a_{k}^{2}+b_{k}^{2}\right)}{4 \omega_{n 1} \omega_{n 2}} \delta^{2}+4 k^{2} \frac{d_{1} d_{2}}{\left(d_{1}+d_{2}\right)^{2}} \Delta \Omega^{2} \leq 0
$$

where the Fourier coefficients $a_{k}$ and $b_{k}$ are defined in (6.11). To carry out numerical calculations we set $n=1, \varphi(\tau)=\cos \tau, d_{1}=d_{2}=1, \omega_{n 1}=1 s^{-1}, \omega_{n 2}=\sqrt{5} s^{-1}$, $l^{2} m=\pi^{2} / 4 \mathrm{~kg} \mathrm{~cm}$, and $r^{2}=4 / \sqrt{5} \mathrm{~cm}^{2}$. The boundary of the combination resonance domain (with $k=1$ ), given by (7.7) in the first approximation, is shown in Fig. 8 (solid curves). The same quantity obtained by numerical evaluation of the monodromy matrix for various values of the parameters $\gamma, \delta$, and $\Omega$ is also shown (dashed curves). The Runge-Kutta method was employed to integrate equation (6.1) with (7.4), (7.5). It is seen from Fig. 8 that the exact (obtained numerically) and approximate (obtained asymptotically) 
domains of the combination resonance are well matched up to the amplitudes $\delta \approx 0.8$.

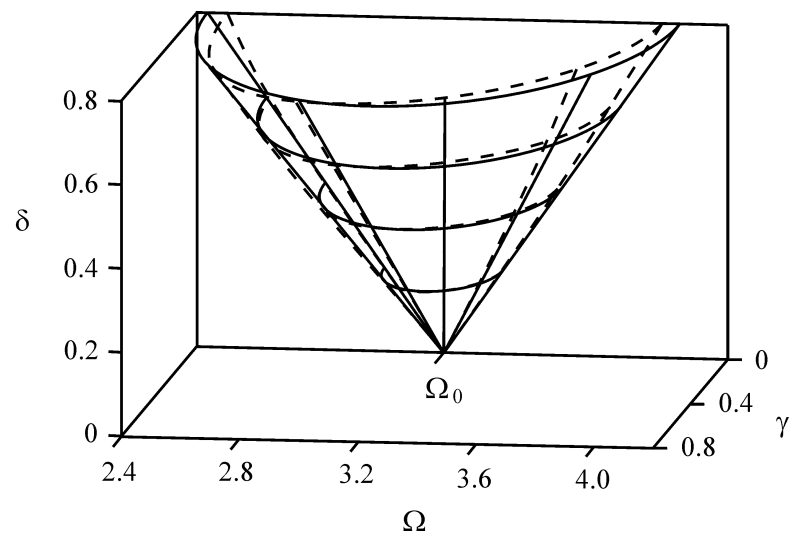

Figure 8: Combination resonance domain for Bolotin's problem.

Other mechanical examples concerning determination of the parametric resonance domains of elastic columns of non-uniform cross-section loaded by longitudinal periodic forces, as well as column optimization problem are presented in Seyranian, Solem and Pedersen (1999, 2000), Mailybaev and Seyranian (2001), and Seyranian and Mailybaev (2003).

\section{Conclusion}

We have considered parametrically excited systems with one and multiple degrees of freedom depending on parameters. First and second order derivatives of the Floquet matrix with respect to parameters are presented in terms of matriciants of the main and adjoint problems and derivatives of the system matrices. With the use of multiparameter perturbation technique for two important cases of excitation matrix: a symmetric matrix, and a stationary matrix, multiplied by a scalar periodic function, the main and combination resonances are studied. It is shown that in both cases the resonance domains are halves of cones in the three-dimensional space of damping magnitude, excitation amplitude and frequency with the boundary surface coefficients depending only on the eigenfrequencies and eigenmodes of the unperturbed conservative system, and the excitation and damping matrices. It turns out that the main and combination resonances can be treated similarly. The obtained relations allow to analyze influence of small damping parameters, as well as growing eigenfrequencies and resonance number on resonance domains. It is shown that the obtained results can be effectively applied to a wide class of mechanical systems to evaluate the parametric resonance domains. Another important application of the given results is connected with the study of stability of periodic solutions in nonlinear dynamical systems.

\section{Acknowledgement}

This research is supported by INTAS grant no. 06-1000013-9019.

\section{References}

Arnold, V.I. (1978). Mathematical Methods of Classical Mechanics. New York: Springer. Bolotin, V.V. (1964). The Dynamic Stability of Elastic Systems, Holden-Day, San Francisco. Bolotin, V.V. (1995). Dynamic stability of structures. In: Non-linear Stability of Structures. Theory and Computational Techniques (Kounadis, A.N. and Kratzig, W.B., eds.). Wien: N.Y.: Springer, 3-72.

Cattani, C., and Seyranian, A.P. (2005). The regions of instability of a system with a periodically varying moment of inertia. J. Appl. Math. Mech. 69 (6): 810-815.

Kapitsa, P.L. (1951). Dynamic stability of a pendulum with vibrating suspension point. 
J. Exp. Theor. Physics. 21 (5): 588-597.

Mailybaev, A.A., and Seyranian, A.P. (2001). Parametric resonance in systems with small dissipation. J. Appl. Math. Mech. 65 (5): 755-767.

Merkin, D. (1997). Introduction to the Theory of Stability. New York: Springer.

Nayfeh, A.H., and Mook, D.T. (1979). Nonlinear Oscillations. New York: John Wiley and Sons.

Panovko, Ya.G., and Gubanova, I.I. (1987). Stability and Vibrations of Elastic Systems. $4^{\text {th }}$ edition. Moscow: Nauka.

Seyranian, A.A., and Seyranian, A.P. (2006). The stability of an inverted pendulum with a vibrating suspension point. J. Appl. Math. Mech.. 70 (5): 754-761.

Seyranian, A.P. (2001). Resonance domains for the Hill equation with allowance for damping. Physics-Doklady 46 (1): 41-44.

Seyranian, A.P. (2004). The swing: parametric resonance. J. Appl. Math. Mech. 68 (5): 757-764.

Seyranian, A.P., and Elishakoff, I. (eds.) (2002) Modern Problems of Structural Stability. Wien: N.Y.: Springer.

Seyranian, A.P., and Mailybaev, A.A. (2001). Parametric resonance in systems with weak dissipation. Doklady Physics 46 (6): 434-439.

Seyranian, A.P., and Mailybaev, A.A. (2003) Multiparameter Stability Theory with Mechanical Applications. New Jersey, London: World Scientific.

Seyranian, A.P., Solem, F., and Pedersen, P. (1999). Stability analysis for multi-parameter linear periodic systems. Archive of Applied Mechanics 69: 160-180.

Seyranian, A.P., Solem, F., and Pedersen, P. (2000). Multi-parameter linear periodic systems: sensitivity analysis and applications. Journal of Sound and Vibration 229: 89-111.

Seyranian, A.P., Yabuno, H., and Tsumoto, K. (2005). Instability and periodic motion of a physical pendulum with a vibrating suspension point (theoretical and experimental approach). Doklady Physics 50 (9): 467-472.

Stephenson, A. (1908). On a new type of dynamical stability. Mem. Proc. Manchester Lit. Phil. Soc. 52 (8): 1-10.

Thomsen, J.J. (1997). Vibrations and Stability. Order and Chaos. London: McGraw-Hill.

Yakubovich, V.A., and Starzhinskii, V.M. (1987). Parametric Resonance in Linear Systems. Moscow: Nauka. 